\title{
Deep Learning Based Iris Recognition System
}

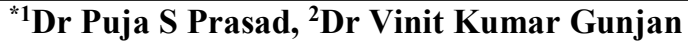 \\ ${ }^{1,2}$ Computer Science \& Engineering Department, CMR Institute of Technology, Medchal, Hyderabad \\ Email: puja.s.prasad@gmail.com
}

\section{Received: $3^{\text {rd }}$ April 2018, Accepted: $1^{\text {st }}$ June 2018, Published: $30^{\text {th }}$ June 2018}

\begin{abstract}
The main focus on this paper is to use multiple instance of a single biometric traits that integrates the left and right iris of a person for authentication. Deep structured learning or we say trained deep learning approach is proposed whose architectural design is based on the combination of and Softmax classifier and Convolutional Neural Network (CNN) for the extraction of distinct floral features from the input iris images. In this work, for learning rate adaptation and weights updating a selective training scheme of CNN that is based on a combination of mini-batch Ada Grad optimization method and backpropagation algorithm and is being proposed. Two databases are used for evaluating performance of this proposed system. The results outputted from the work of this proposed system outperform achieved an identification rate Rank-1 of $99.8 \%$ on all the corresponding different databases that is used here as well as recognition time is also reduces.
\end{abstract}

Keywords: Iris, Unimodal biometric, Ada Grad optimization, Convolutional Neural Network, Backpropagation algorithm.

\section{Introduction}

For authentication of an individual person biometric systems are growing rapidly and also it is a challenging technologies that can be used for identification and verification of a person without the use of traditional method for authentication like Cards, passwords, IDs. By this reason, iris recognition for authenticating a person has been used in many useful applications, authenticating a person for accessing in sensitive area, airport access, database access national identification cards, as well as financial services and is said to be unique as well as best reliable and accurate biometric systems. Multimodal biometric systems have been used widely in many areas for authentication purposes realworld applications. The limitations present in unimodal system like noise, non-universality, intra-class variability, inter-class variability, population coverage, and vulnerability to spoofing. In our work, multimodal system is given which is actually based on the concept of deep learning for representations of left and right irises of a person, and after that the features obtain from both the left and right irises are fused by a method name called ranking-level fusion Numerous studies have demonstrated that the iris features have a very unique characteristics compare to other biometric traits (e.g., palm print, earlobe, DNA, fingerprint), which make it highly demanding for application where high reliability as well as more accurate decision required $[6,9,10]$.

The iris feature consists of circular annular region of the eye which lie present in between the white sclera and black pupil; Due to this it is completely safe from changing external environments. It is also observed that the in eye the iris provides uniqueness and richness to very high degree, that is the reason it very rarely that any two iris features or their patterns to be the same. Irises of children from same parents or identical twins, or from the left and right iris of an individual person are unlikely to be same. This distinctiveness in iris feature patterns is due to the presence of different pattern inside it like crypts, rings, ridges, furrows, zigzag patterns, freckles etc. [1]. Iris provides very high stability during person's whole life until some accident occurs. Moreover, it is observed that the iris is the most reliable biometric trait compares to other against spoofing attacks and deceitful methods as well as by a to change its patterns by surgery is very unsafe, however in case of fingerprint which is relatively easily tamper. In spite of number these advantages, iris method of authenticating person and building that system is considered a very challenging problem due to the reason of presence of non-useful parts, such as pupil, eyelashes, eyelids, and specular reflection's during image acquisition process which may highly affect the iris segmentation and recognition results. Broadly, there are two types of biometric called multimodal biometric systems. Unimodal systems use only single biometric trait like fingerprint, face, left or right iris to authenticate certain person's identity. Even though, unimodal biometric system has been widely used in sensitive applications and government agencies with a very high level of safety, they itself suffers from a number of limitations as well as problems that can entirely affect the performance as well as reliability $[3,4,5]$. These critical problems or limitations are: 
- noises present in the sensed biometric trait

- intra-class variations

- non-universality

- inter-class variations

- vulnerability to spoof attacks

These are the limitations of unimodal biometric systems can be handled uniting two or more features from different biometric traits for authenticating a person individuality and this is the base of multimodal biometric. Now a days more attention has been concentrated on multimodal biometric systems due to their more accurate results to attain improved performance compared to unimodal systems. Multimodal systems can cover enough population by efficiently solving the problem of non-universality during enrolment phase. In addition, these systems can also provide a higher accuracy as well as greater confrontation to unauthorized user or imposter than unimodal systems. More details regarding addressing the different issues arises due to unimodal system can be found in the given references. In general, designing a multimodal system as well as its implementation is stimulating as well as challenging. Large number of factors that greatly affect the overall performance of the multi biometric system need to be focused, which includes cost, accuracy, different algorithm as well fusion and matching methods employed. However, the main focus in multimodal biometric system is the selection of biometric trait as well as selection of different fusing algorithm and matching algorithm [11]. For different multimodal biometric systems, if the biometric system works in identification mode of recognition, then the output obtained from the classifier can be mark as a ranks list of the registered or enrolled subjects. Rank list is a set of all possible matches which is actually placed or sorted in sinking or descending order of confidence. Ranking level fusion methods can be applied to combine the ranks formed by each separate classifier in order to believe a consent rank for each person. Finally, output called the scores are sorted in decreasing order and the individuality of a person with score having lower value is presented as authentic person [2]. In our work, we are going to use two different learning techniques which is the combination of Softmax and CNN. The importance of convolutional nets (ConvNets or CNNs) in image recognition is one of the significant reasons why the world has woken up to the effectiveness of deep learning. They are driving towards major advances in computer vision $(\mathrm{CV})$, which has pure applications for drones, robotics, security, medical diagnoses, selfdriving cars as well as treatments for the visually impaired. Moreover, Convolutional Neural Network have a number of advantages in contrast to other DNNs, like simpler architecture, adaptability, and fewer free parameters fast convergence. Besides these
CNNs are invariant to image twists, such as translation, rotation and scaling. The Classifier Softmax is a discriminative classifier mainly used for different multi-class classification purposes. Multi -Class Classification using classifier softmax have been selected on priority over other $\mathrm{CNN}$ because it gives very good results in contrast to other different standard classifiers, like Support Vector Machines (SVMs) in terms of speed and accuracy.

The aim of this work can be described as follows:

- An authentication system called multimodal biometric which uses two instances of eye is proposed which is more efficient and robust by fusing features of both left and right iris.

- A well-organized model called Deep learning called IrisConv is used whose design is based on a mixture of a Softmax and $\mathrm{CNN}$ classifier to extract discriminative features from the iris image without any need of domain knowledge and classify it into one of $\mathrm{N}$ classes.

- Based on our knowledge there are very few work to explores the potential use of $\mathrm{CNNs}$ for the iris recognition system, that works in the identification mode

- The overall act of the our proposed system is experienced on two different public datasets: and IITD and CASIA-IrisV3 Interval iris databases. The outcomes obtained from this dataset are have checked that the proposed system perform well compare to other approaches like Scattering transform, Wavelet transform, PCA.

\section{Related Works and Motivations:}

John Daugman the main person who give iris characteristic develop a first model of an iris identification system. Daugman's iris system is very popular marketable iris recognition systems used in various organization for authentication using iris. Daugman's iris system works on different stages such as segmentation, followed by normalization, then feature encoding and iris code matching. Wildes et al." [1994] design and implemented an automated recognition system for iris, in which first localization of iris region is done by using histogram processing, and then image filtering with the band pass filters which is isotropic done. After that "Wildes et al." [1996] again proposed a model for personal authentication using iris called machine vision system for iris recognition. As iris is an overt body its appearance is agreeable to remote examination with the help of a machine-vision system. This system exhibits perfect performance in the evaluation of 520 iris images. "Wildes." [1997] proposed another modal that 
uses diffuse light source for personal identification system using iris. In this modal he first extracted the boundaries of iris by edge detection method called canny edge detection then it is followed by Hough transform circular function. By the use of Laplacian Gaussian filter at multiple scale, he produced the templates using and in then in matching stage, he computed the correlation.

Boles and Boashash [1998] calculated a new technique by using different resolution levels by using zerocrossings of wavelet transform in different concentric circle of iris, and then the 1Dimensional signals for an iris recognition system for identification by using iris images and their wavelet transform. The matching was obtained by taking into account two variation functions between the iris image and iris template. Zhu et al. [2000] also works on extraction of global feature for personal identification using iris by applying Gabor filter and wavelet transform technique, by applying the multi-channel Gabor filter and wavelet transform technique. As global feature is less sensitive to noise. El-Bakry et al. [2001] proposed the recognition system using iris which is fast by using neural networks. Lim et al [2008] proposed an algorithm name, Efficient Iris Recognition system through Improvement of Feature Vector and Classifier. Segmentation, normalization and encoding feature method was taken same as the Daugman techniques. The matching stage, has two methods of learning called winner selection and weight vector initialization for vector quantization (LVQ) and this was used to categorize the feature vectors. This system actually covered the verification and identification of individual's personality. Ma L. et al. [2000] employed another type called circular symmetric filters (CFS) for iris recognition system. CFS or circular symmetric filters has certain variation from the Gabor wavelet used a function called Circular symmetric sinusoidal function for modulation. To detect boundaries, they use different edge detection function and Hough transform function. This system, focused the fact that centre point coordinate of the pupil and iris was generally not the same. "SanchezAvila et al." [2003] used wavelet transform called dyadic wavelet transform. They compared the results for different distances technique i.e. Euclidean, hamming and the direct distance using zero-crossing, for classification and verification and they come at the point that Hamming distance had better results. The iris recognition systems that are proposed by Daugman and Wildes [1994] were intended for both verification and identification mode but the remaining were proposed for identification.

\section{Overview of the Proposed Approaches}

In this part, a brief description of the proposed Deep Learning approach is defined, which uses two different learning techniques: Convolutional Neural Network and Softmax Classifier. The main goal here is to examine their inside structures and identify their pros and cons to and then integrates the strength of this two techniques to the proposed iris recognition system.

\subsection{Convolutional Neural Network}

$\mathrm{CNN}$ is a feed forward network in which each input is received from neurons after that it performs, a dot product and that are optionally follows with a nonlinearity. The whole network gives a single differentiable score function: by using raw image pixels on one end to at the other class scores.

$\mathrm{CNN}$ architecture have several different set of layer which include convolutional layer sets that are locally connected, a pooling layer and also a completely connected layer [6]. These architectural concept males $\mathrm{CNN}$ very popular in different field like image processing, pattern recognition and natural language processing. Each neuron accept input from the small region called local perspective region. After that elementary features like edges, ridge, curve are extracted which play a very important role to reduce intra class variation and increase inter class variation. In order to reduce complexity convolutional layer, apply parameter sharing. To reduce the spatial size of the output non -linear down sampling applied[16,18].

\section{The Proposed System}

The initial step in the proposed system is the preprocessing stage in which iris image is separated from the irrelevant background feature like sclera, pupil, eyelashes etc. Instead of taking whole eye as input only iris is taken to reduce the complexity of input. After localization iris image go into the normalization process and CNN is used as feature extractor. Softmax Classifier is used to obtain matching score from the connected neuron. Now matching score from both the iris is fused to identify the subject. Different CNN configurations are trained during training and the training set and finally tested on validation set to obtain the best one with the minimum error that we call ConvNetIris.

\subsection{Training Methodology}

This proposed methodology is performed on a specific set of data in which data are divided into two different set one for training and other for testing. The given training set is divided into three set that starts training at particular $\mathrm{CNN}$ configuration and another set is used for testing purposes. Unlike neural network $\mathrm{CNN}$ consists of three dimensions where neurons are arranged in respective three dimension called width, height and depth.

The main phases of the given proposed training steps are as follows: 
1. Splitting the proposed system dataset into three different sets: called Training another one Validation and last one Test set. The database from two different database set are divided into three sets for training validation and testing.

2. Selection of a CNN architecture as well as set of training Parameters-Training parameters are selected as well as different $\mathrm{CNN}$ architecture is also selected.

3. After that training of each Convolutional Neural Network configuration using the training set is done.

4. Evaluation of each Convolutional Neural network configuration is done using the validation set.

5. Reiterate steps 3 through 4 using $N$ number of times. 6. After that Selection of best $\mathrm{CNN}$ configuration that have minimal error on the validation set.

7. Finally evaluation of the best Convolutional Neural Network configuration using the test set.

\section{Conclusion}

In the above proposed paper multimodal biometric method for authentication is proposed by using the both irises as a biometric. A deep learning method is proposed which uses CNN and Softmax as feature extractor. The deep learning structure which is in fact based on a combination of the both Softmax classifier and $\mathrm{CNN}$. As iris consists of rich feature this technique is used to extract the features of both left and right irises without any need of domain knowledge. After that it is classified into classes. After that Identification and ranking scores are calculated for each individual. For learning rate adaptation and weights updating a selective training scheme of CNN that is based on a combination of mini-batch Ada Grad optimization method and back-propagation algorithm and is being proposed. Two databases are used for evaluating performance of this proposed system. The outcome obtained from the work of proposed system outperform achieved a Rank-1 identification rate which is equal to $99.8 \%$ on all the corresponding databases that is used here as well as recognition time is also reduces.

\section{References}

1. A. Baig, A. Bouridane, F. Kurugollu, and G. Qu, "Fingerprint-iris fusion based identification system using a single hamming distance matcher," International Journal of Bio-Science and Bio-Technology, vol. 1, no. 1, pp. 47-58, 2009. View at Google Scholar · View at Scopus

2. Boles, W. W. B. B. [1998]. A Human Identification Technique Using Images of the Iris and Wavelet Transform. IEEE Transactions on Signal Processing. 46: 1185-1188 Wayman, J. [2014]. Handbook of Iris Recognition. Biometrics, IET. 3: 41-43.

3. Browning, K. and Orlans, N. [2014]. Biometric Aging.Daugman, J. and Downing, C. 2013. No
Change Over Times Shown in Rankin et al. Iris Recognition Failure Over Time: The Effects Of Texture. Pattern Recognition. 46: 609-610. Asker

4. Boles, W. W. B. B. [1998]. A Human Identification Technique Using Images of the Iris and Wavelet Transform. IEEE Transactions on Signal Processing. 46: 1185-1188.

5. Boyce, C. K., and Hornak, L. A. [2007]. On Techniques for Angle Compensation in Nonideal Iris Recognition. IEEE Transactions on Systems, Man, and Cybernetics, Cybernetics. (B)37: 11761190 .

6. Bazama, A. E., and Hassan, Y. F. [2012]. Hybrid System of Cellular Automata, PCA and Support Vector Machine for Noise Reduction and Classification in Human Iris Recognition. IJIIP: International Journal of Intelligent Information Processing. 3: 47-67.

7. Ballan.M, [1998] "Directional Fingerprint Processing", International Conference on Signal Processing, vol.2, pp. 1064-1067

8. Bodade, R. M., and Talbar, S. N. [2009]. Shift invariant iris feature extraction using rotated complex wavelet and complex wavelet for iris recognition system. Seventh International Conference on Advances in Pattern Recognition. 449-452.

9. Besbes F., Trichili H., Solaiman B. Multimodal biometric system based on fingerprint identification and iris recognition. Proceedings of the 3rd International Conference on Information and Communication Technologies: From Theory to Applications (ICTTA '08); April 2008; Damascus, Syria. IEEE; pp. 1-5.

10. Chandran GC, Rajesh RS (2009). Performance Analysis of Multimodal Biometric System Authentication, Int. J. Computer. Sci. Network Security, 9: 3.

11. Dehkordi, A. B. and Abu-Bakar, S. A. R. [2015]. Adaptive Fuzzy Switching Noise Reduction Filter for Iris Pattern Recognition. Jurnal Technology. 73: 11 .

12. Duresuoquian Miao, Qingshi Tang, and Wenjie Fu [2007], "Fingerprint Minutiae Extraction Based on Principal Cures", the Journal of the Pattern Recognition Letters, vol. 28, pp. 2184-2189

13. Daugman, J. [2009] Iris Recognition at Airports and Border-Crossings. Encyclopedia of Biometrics. ed: Springer. 819-825.

14. Daugman, J. [2004]. How Iris Recognition Works. Circuits and Systems for Video Technology, IEEE Transactions on. 14: 21-30.

15. Daugman, J. [2007]. New Methods in Iris Recognition. Systems, Man, and Cybernetics, Part B: Cybernetics, IEEE Transactions on. 37: 11671175.140-144. 
16. Dozier, G., Bell, D., Barnes, L., and Bryant, K. [2009]. Refining Iris Templates via Weighted Bit Consistency. Proc. Midwest Artificial Intelligence and Cognitive Science (MAICS) Conference. 1-5.

17. Dozier, G., Frederiksen, K., Meeks, R., Savvides, M., Bryant, K., Hopes, D. [2009]. Minimizing the Number of Bits Needed for Iris Recognition via Bit Inconsistency and Grit. IEEE Workshop on Computational Intelligence in Biometrics: Theory, Algorithms, and Applications (CIB 2009). 30-37.

18. Da Costa, R. M., and Gonzaga, A. [2012]. Dynamic Features for Iris Recognition. IEEE Transactions on Systems, Man, and Cybernetics, Part B: Cybernetics. 42: 1072-1082

19. Dutta, M. K., Gupta, P., and Pathak, V. K. [2009]. Biometric Based Unique Key Generation for Authentic Audio Watermarking. Pattern Recognition and Machine Intelligence. Springer. 458-463.

20. Feifei cui, Gongping yang, [2011] "Score level fusion of fingerprint and finger vein Recognition", Journal of Computer Information's systems: 16, 5723-5731, 2011 